\title{
Compromised gastrointestinal integrity in pigtail macaques is associated with increased microbial translocation, immune activation, and IL-17 production in the absence of SIV infection
}

\author{
NR Klatt $^{1}$, LD Harris ${ }^{1}$, CL Vinton ${ }^{1}$, H Sung ${ }^{2}$, JA Briant ${ }^{1}$, B Tabb ${ }^{3}$, D Morcock ${ }^{3}$, JW McGinty ${ }^{1}$, JD Lifson ${ }^{3}$, \\ BA Lafont ${ }^{1}$, MA Martin ${ }^{1}$, AD Levine ${ }^{2}$, JD Estes ${ }^{3}$ and JM Brenchley ${ }^{1}$
}

Pigtail macaques (PTMs) rapidly progress to AIDS after simian immunodeficiency virus (SIV) infection. Given the strong association between human immunodeficiency virus (HIV) and SIV disease progression and microbial translocation and immune activation, we assessed whether high basal levels of immune activation and microbial translocation exist in PTMs. We found that before SIV infection, PTMs had high levels of microbial translocation that correlated with significant damage to the structural barrier of the gastrointestinal tract. Moreover, this increased microbial translocation correlated with high levels of immune activation and was associated with high frequencies of interleukin-17-producing $T$ cells. These data highlight the relationship among mucosal damage, microbial translocation and systemic immune activation in the absence of SIV replication, and underscore the importance of microbial translocation in the rapid course of disease progression in SIV-infected PTMs. Furthermore, these data suggest that PTM may be an ideal model to study therapeutic interventions aimed at decreasing microbial translocation-induced immune activation.

\section{INTRODUCTION}

The mucosal immune system is intricate and complex, and relies on a balance of multiple cell types to maintain homeostasis and to function appropriately. ${ }^{1,2}$ The tight epithelial barrier of the gastrointestinal (GI) tract functions to prevent commensal organisms and pathogens from crossing the intestinal lumen into circulation, while allowing nutrients to be absorbed. ${ }^{3} \mathrm{An}$ important role for mucosal $\mathrm{T}$ cells in maintaining GI tract function has recently been highlighted by numerous studies dedicated to a newly identified subset of CD $4+\mathrm{T}$ cells, Th17 cells, which are found most prominently in the GI tract. ${ }^{4-10}$ These $\mathrm{T}$ cells produce interleukin 17 (IL-17), which is important in adaptive immunity against extracellular bacteria and fungi, recruit neutrophils, induce defensin production, promote enterocyte homeostasis, and are important for maintenance of epithelial tight junctions in mucosal tissues. ${ }^{11-16}$ However, Th17 cells have also been implicated as potent inducers of tissue inflammation in several GI disorders such as Crohn's disease and ulcerative colitis, which result from a chronic inflammatory state associated with infiltration of inflammatory immune cells and damage to the gut epithelium. ${ }^{17-21}$ Specifically, an increased frequency of IL-17-producing cells in the GI tract has been directly associated with inflamed mucosa in inflammatory bowel diseases. ${ }^{13,19,20,22,23}$ Thus, dysregulation of mucosal-associated lymphoid tissues (MALT) can lead to pathogenic consequences.

Dysfunction of the mucosal immune system has also been shown in chronic viral infections, particularly during human immunodeficiency virus (HIV)/simian immunodeficiency virus (SIV) pathogenesis. HIV and SIV infections are characterized by chronic virus replication and depletion of $\mathrm{CD} 4+\mathrm{T}$ cells, ultimately resulting in opportunistic infections and progression to AIDS. Because HIV/SIV preferentially infects CD4 + CCR5 + $\mathrm{T}$ cells, which are enriched in mucosal tissues, there is a rapid and severe depletion of CD4 + T cells in MALT during acute infection. ${ }^{24-28}$ However, CD4 depletion alone is not sufficient to cause AIDS. ${ }^{26,29-31}$ Rather, the strongest predictor of disease progression is the extent of chronic, systemic immune 
activation associated with HIV pathogenesis. ${ }^{32}$ This systemic immune activation is characterized by increased lymphocyte proliferation, high rates of lymphocyte apoptosis, cell cycle dysregulation, and increased levels of proinflammatory cytokines. ${ }^{33-35}$ Massive infection of CD4 + T cells in MALT early in HIV/SIV infections is directly associated with inflammation and breakdown of the mucosal integrity; this allows microbial products to translocate from the lumen of the GI tract into the peripheral circulation..$^{25,36-42}$ Translocation of microbial products during HIV/SIV infections, shown by an increase in plasma lipopolysaccharide (LPS) and bacterial DNA levels, is associated with systemic immune activation. ${ }^{25,36-41}$ The GI pathology associated with HIV/SIV infections includes significant enterocyte apoptosis, enteropathy of the MALT, as well as increased levels of inflammation and decreased levels of mucosal repair and regeneration. ${ }^{43-45}$ However the mechanisms underlying the damage to the GI tract are not well understood, and clarifying to what extent structural and/or immunological damage to the GI tract and microbial translocation underlies immune activation is vital to characterize the interactions between the MALT and peripheral immune system.

Nonhuman primate models are essential to better understand how and to what extent dysfunction and damage to the mucosal immune system affects systemic immune activation in vivo. Pathogenic SIV infection of Asian rhesus macaques (RMs; Macaca mulatta) is the most widely studied nonhuman primate model for HIV pathogenesis to date. In this model, infection of RM with SIVmac strains (derived from SIVsmm) recapitulates many key features of HIV infection in humans, ${ }^{46-48}$ including rapid and severe depletion of $\mathrm{CD} 4+\mathrm{T}$ cells in mucosal tissues during acute infection, progressive loss of CD $4+\mathrm{T}$ cells in periphery during chronic infection, high viral load, high levels of immune activation, and microbial translocation. $33,36,46,47,49,50$ Another important model for HIV infection is SIV infection of pigtail macaques (PTMs; M. nemestrina). Similar to RMs, after SIV infection, PTMs lose CD4 + T cells, have high levels of immune activation, and progress to AIDS. ${ }^{51-53}$ These animals are of particular interest, in that PTMs typically progress to AIDS more rapidly than RMs. ${ }^{54,55}$ After infection with SIVsmE543, the majority of PTMs progress to AIDS within 6 months after infection (as opposed to $\sim 2$ years for RMs). ${ }^{54,55}$ This rapid disease progression observed in PTMs is not associated with virus inoculation, but is likely due to host factors. ${ }^{55-57}$ Interestingly, uninfected PTMs in captivity have an increased incidence of diarrhea and GI diseases, and older animals frequently present with systemic amyloidosis. ${ }^{58,59}$ Indeed, in 47 SIV-uninfected PTMs in our animal facility that have been followed up for a minimum of 5 years, 24 animals (51.1\%) have had histories of GI disorders, as defined by at least two bouts of diarrhea requiring treatment. In contrast, only 12 of 103 (11.6\%) SIV-uninfected RMs in our facility have had such disorders $(P<0.0001)$. Similar observations have also been made at two separate animal facilities across the world (Shiu-Lok Hu, University of Washington, and Stephen J Kent, University of Melbourne; personal communication). Moreover, after certain SIV infections, such as SIVPBj-14 infection, PTMs are more susceptible than RMs to death resulting from GI distress. ${ }^{60}$ We hypothesized that the rapid disease progression observed in PTMs after SIV infection may, in part, be due to preexisting conditions that result in dysfunction and damage of the mucosal immune system, leading to increased microbial translocation and consequent immune activation. To address this hypothesis, in this study, we assessed the T cell immunophenotype, function, and activation in multiple anatomical sites including blood, lymph nodes (LN), and tissues of the GI tract. We also determined the level of mucosal integrity, microbial translocation, and immune activation in SIV-uninfected PTMs in comparison with RMs.

\section{RESULTS \\ Uninfected PTMs have a higher frequency of proliferating and memory-effector T cells than RMs}

We initially performed flow cytometric analysis to study cellular markers associated with activation and differentiation in a cohort of SIV-uninfected PTMs and RMs. We found that PTMs had significantly increased frequencies of proliferating CD4 + Ki67 + T cells in blood compared with RMs (10.62 $\pm 4.5 \%$ compared with $4.59 \pm 1.7 \%, P=0.0006$; Figure 1a), as well as increased CD8 $+\mathrm{Ki} 67+\mathrm{T}$ cells in blood from PTMs compared with RMs $(10.06 \pm 5.9 \%$ compared with $5.76 \pm 1.8 \%, P=0.0637$; Figure 1b). Accordingly, PTMs also had a significantly greater frequency of memory and the frequency of effector $\mathrm{T}$ cells in blood as compared with RMs, as measured by CD28 + CD 95 + and CD28 - T cells. On average, $72.02 \%( \pm 12.0)$ of CD $4+\mathrm{T}$ cells were of memoryeffector phenotype in PTMs, as compared with $47.97 \%( \pm 18.9)$ of RM CD $4+$ T cells being memory-effector cells $(P=0.0013$; Figure 1c). A similar finding was observed for CD8 $+\mathrm{T}$ cells, as PTMs had an average of $80.34 \%( \pm 16.3)$ of CD $8+$ memory $\mathrm{T}$ cells, as compared with $66.34 \%( \pm 14.5)$ of RM CD8 + T cells having a memory-effector phenotype $(P=0.0112$; Figure 1d).

High frequencies of activated memory-effector $\mathrm{T}$ cells in peripheral blood may not reflect frequencies of $\mathrm{T}$ cells in all anatomical sites. ${ }^{43,61}$ Therefore, we measured these cells by flow cytometry carried out on mononuclear cells isolated from multiple tissues obtained from killed, SIV-uninfected PTMs and RMs. We found high frequencies of memory CD4 + T cells in multiple PTM tissues (Figure 1c), with an average of $87.74 \%( \pm 7.8)$ in spleen, $78.77 \%( \pm 12.4)$ in axillary $\mathrm{LN}, 78.15 \%( \pm 9.9)$ in inguinal $\mathrm{LN}, 73.97 \%( \pm 13.8)$ in mesenteric LN, $85.36 \%( \pm 18.2)$ in duodenum, $93.38 \%( \pm 4.3)$ in jejunum, $85.02 \%( \pm 12.4)$ in ileum, $91.57 \%$ $( \pm 5.0)$ in cecum, and $88.63 \%( \pm 10.2)$ in colon. In comparison, RMs had lower levels of CD4 + memory-effector T cells in many tissues (Figure 1c), with an average of $77.23 \%( \pm 13.4)$ in spleen, $49.28 \%( \pm 7.8)$ in axillary LN, $50.42 \%( \pm 6.3)$ in inguinal $\mathrm{LN}$, $36.75 \%( \pm 10.23)$ in mesenteric LN, $97.47 \%( \pm 4.3)$ in duodenum, $91.16 \%( \pm 7.9)$ in jejunum, $63.92 \%( \pm 10.6)$ in ileum, $66.32 \%$ $( \pm 6.0)$ in cecum, and $62.52 \%( \pm 10.1)$ in colon. Indeed, the frequency of memory-effector CD4 + T cells in PTMs was significantly higher than in multiple tissues from RM (Figure 1c), including spleen $(P=0.0218)$, axillary $\mathrm{LN}(P=0.0010)$, inguinal LN $(P=0.0004)$, mesenteric LN $(P=0.0026)$, ileum $(P=0.0076)$, cecum $(P=0.0007)$, and colon $(P=0.0016)$. We also found high 
frequencies of memory-effector CD8 + T cells in the same PTM tissues (Figure 1d), with an average of $88.1 \%( \pm 12.9)$ in spleen, $72.44 \%( \pm 21.6)$ in axillary LN, $64.61 \%( \pm 22.4)$ in inguinal $\mathrm{LN}$, $61.27 \%( \pm 23.6)$ in mesenteric $\mathrm{LN}, 93.16 \%( \pm 14.7)$ in duodenum, $98.42 \%( \pm 1.3)$ in jejunum, $79.79 \%( \pm 20.0)$ in ileum, $86.51 \%$ $( \pm 14.2)$ in cecum, and $85.01 \%( \pm 20.4)$ in colon. In comparison, the frequency of CD8 + memory-effector T cells in RM was on average $82.77 \%( \pm 13.6)$ in spleen, $35.67 \%( \pm 9.9)$ in axillary $\mathrm{LN}$, $38.5 \%( \pm 13.6)$ in inguinal LN, $22.52 \%( \pm 7.4)$ in mesenteric LN, $95.67( \pm 2.7)$ in duodenum, $91.16 \%( \pm 8.3)$ in jejunum, $41.4 \%$ $( \pm 14.0)$ in ileum, $45.1 \%( \pm 7.3)$ in cecum, and $49.35 \%( \pm 13.7)$ in colon. The frequency of memory-effector CD8 + T cells was also significantly higher in many tissues from PTMs compared with RMs (Figure 1d), including in axillary LN $(P=0.0017)$, inguinal LN $(P=0.0312)$, mesenteric LN $(P=0.0010)$, ileum $(P=0.0080)$,

a
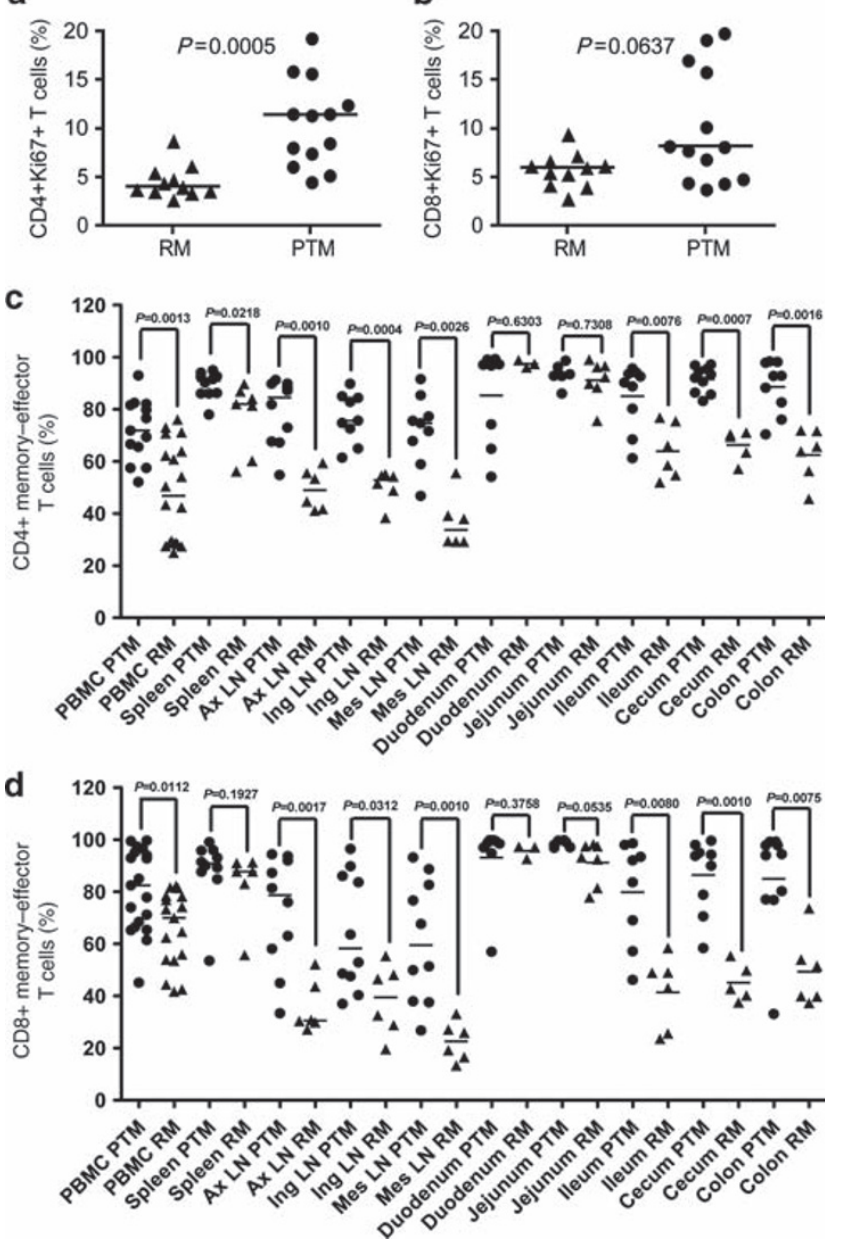

Figure 1 Uninfected pigtail macaques (PTMs) have high levels of immune activation and memory-effector $T$ cells compared with rhesus macaques (RMs). (a) Frequencies of CD4+Ki67 + T cells in blood; (b) frequencies of CD8+Ki67+ T cells in blood; (c) frequencies of CD4+ memory-effector $T$ cells in blood and necropsy tissues; and (d) frequencies of CD8+ memory-effector T cells in blood and necropsy tissues. PTM, circles, RM, triangles. Memory-effector T cells were determined by gating on both CD28 + C95 + and CD28 - subsets, as measured by flow cytometry. Horizontal bars indicate median. $P$-values calculated using Mann-Whitney U-test. Ax LN, axillary lymph node; Ing LN, inguinal LN; Mes LN, mesenteric LN; PBMC, peripheral blood mononuclear cell. cecum $(P=0.0010)$, and colon $(P=0.0075)$. These data are of particular interest, as it is expected that effector tissues of the GI tract have high frequencies of memory and effector T cells, as previously described in RMs and humans. ${ }^{62,63}$ However, lymphoid tissues such as spleen and LN typically have much lower frequencies of memory $\mathrm{T}$ cells and higher frequencies of naïve $\mathrm{T}$ cells, as shown by the low level of memory-effector T cells in RMs (Figure 1c and d). Here, we describe that PTMs have very high frequencies of memory $\mathrm{T}$ cells (and thus low frequencies of naïve T cells) in all sites examined. Moreover, these results highlight the anatomically restricted differences in phenotypic distribution of T cells. Even across different regions of the GI tract, T-cell subsets are not uniform, with differing frequencies of memory and effector $\mathrm{T}$ cells residing in different locations (Figure 1c and d, Supplementary Tables 1 and 2 ). Of note, the increased proliferating and memory-effector $\mathrm{T}$ cells observed is not due to a transient infection, as longitudinal sampling showed that these cells remained at high frequencies over time (data not shown). These data suggest that one of the mechanisms underlying the increased SIV disease progression in PTMs may involve the high baseline frequency of activated targets for the virus before SIV infection.

\section{Uninfected PTMs have a higher frequency of CD4 + CCR5 +} T cells as compared with RMs, with high levels in GI tissues Because HIV and SIV preferentially infects activated CCR5 + memory CD $4+\mathrm{T}$ cells, ${ }^{64}$ we next determined whether the increased frequency of memory and effector $\mathrm{T}$ cells observed in PTMs translated to an increased frequency of CCR5 + $\mathrm{T}$ cells. Thus, we measured the frequency of CCR $5+\mathrm{T}$ cells in peripheral blood and multiple tissues from necropsies of both SIV-uninfected PTMs and RMs. We found that PTMs had significantly higher frequencies of CD $4+$ CCR $5+\mathrm{T}$ cells in blood as compared with RMs ( $14.54 \pm 6.3 \%$ compared with $7.76 \pm 4.8 \%, P=0.0008$; Figure 2), as well as a higher frequency of CD8 + CCR $5+\mathrm{T}$ cells (data not shown). The frequency of CD4 + CCR5 + T cells was elevated in multiple anatomical sites

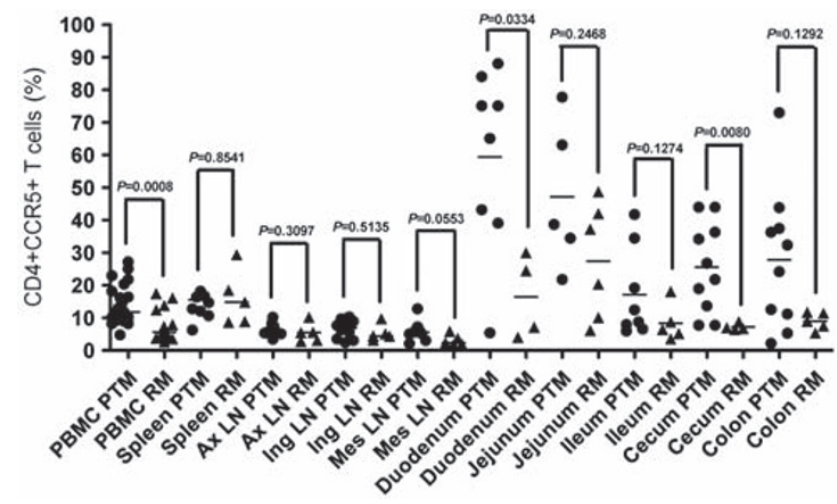

Figure 2 Uninfected pigtail macaques (PTMs) have high frequencies of CD4+CCR5+T cells compared with rhesus macaques (RMs). Frequencies of CD4+CCR5 + T cells in blood and necropsy tissues. PTM, circles, RM, triangles. Horizontal bars indicate median. $P$-values calculated using Mann-Whitney $U$-test. Ax LN, axillary lymph node; Ing LN, inguinal LN; Mes LN, mesenteric LN; PBMC, peripheral blood mononuclear cell. 
of PTMs (Figure 2), with an average of $14.17 \%( \pm 3.7)$ in spleen, $6.41 \%( \pm 1.9)$ in axillary LN, 6.54\% $( \pm 2.8)$ in inguinal LN, 5.97\% $( \pm 2.6)$ in mesenteric LN, $59.4 \%( \pm 28.2)$ in duodenum, $47.18 \%$ $( \pm 22.7)$ in jejunum, $17.17 \%( \pm 13.8)$ in ileum, $25.53 \%( \pm 13.8)$ in cecum, and $27.86 \%( \pm 21.5)$ in the colon of PTMs (Figure $2 \mathbf{b})$. In comparison, the average frequency of CD $4+$ CCR $5+$ T cells in $\mathrm{RM}$ was $16.04 \%( \pm 8.5)$ in spleen, $5.43 \%( \pm 3.0)$ in axillary LN , 5.34\% $( \pm 2.5)$ in inguinal LN, 3.15\% $( \pm 1.7)$ in mesenteric $\mathrm{LN}, 16.42 \%( \pm 12.8)$ in duodenum, $27.43 \%( \pm 17.7)$ in jejunum, $8.38 \%( \pm 5.6)$ in ileum, $7.24 \%( \pm 1.1)$ in cecum, and $8.98 \%( \pm 2.7)$ in the colon (Figure 2). In addition to blood, the frequency of CD $4+$ CCR $5+$ T cells was significantly higher in duodenum $(P=0.0334)$ and cecum $(P=0.0080)$ of PTMs compared with RMs (Figure 2). We also investigated whether CD4 + CCR5 + $\mathrm{T}$ cells had higher levels of CCR 5 per cell, and found that although PTMs had a slightly increased mean fluorescence intensity of CCR5 on CD4 + T cells, the difference was not significant between PTMs and RMs $(P=0.1008$, data not shown). Not all tissues had significantly higher frequencies of CD4 + CCR5 + T cells in PTMs compared with RMs, likely because of the low numbers of RMs available for analysis, but Figure 2 shows that on average, PTMs have significantly more CD $4+$ CCR $5+$ T cells than RMs. Of note, there was a greater frequency of CD4 + CCR5 + T cells in the duodenum and jejunum compared with other tissues, including the large intestine (Supplementary Table 3). These data show that before SIV infection, PTMs have an increased frequency of activated targets for SIV infection, which may, in part, explain the rapid disease progression observed after SIV infection, as increased targets may result in more rapid CD4 + T-cell depletion.

\section{Uninfected PTM T cells are enriched for IL-17 but not for interferon- $\gamma$ production when compared with RM, and IL-17+ T cells are found in all tissues of PTM}

To determine the functionality of T cells in PTM and RM and to attempt to understand the mechanisms underlying the increased frequencies of activated and memory T cells in PTM, we stimulated PBMCs with the mitogens phorbol myristate acetate and ionomycin, and measured interferon- $\gamma$ (IFN $\gamma$ ) and IL-17 production by flow cytometry. IFN $\gamma$ can be produced by $\mathrm{T}$ cells in all tissues, typically in response to intracellular pathogens such as viruses. In contrast, IL-17, is produced by $\mathrm{T}$ cells in response to extracellular bacteria and fungi, and IL-17-producing $\mathrm{T}$ cells are found at high frequencies in mucosal tissues in which these antigens predominate. ${ }^{65}$ Surprisingly, we found that PTMs had significantly lower frequencies of CD4+ T cells that produce IFN $\gamma$ in peripheral blood compared with RMs (15.1 $\pm 7.7 \%$ compared with $38.93 \pm 21.3 \%, P=0.0095$; Figure 3a). However, there was no difference between the frequencies of IFN $\gamma$-producing CD8 + T cells $(44.64 \pm 24.3 \%$ compared with $39.4 \pm 18.2 \%, P=0.6$; Figure 3 b). Interestingly, PTMs had higher frequencies of peripheral blood CD $4+\mathrm{T}$ cells that produced IL-17 (5.92 $\pm 3.3 \%$ compared with $1.91 \pm 1.8 \%$, $P=0.0081$; Figure 3c). Perhaps most intriguingly, PTMs also had higher frequencies of CD8 + T cells that produced IL-17 (3.33 $\pm 1.8 \%$ compared with $0.11 \pm 0.2 \%, P<0.0001$; Figure $3 d$ ).
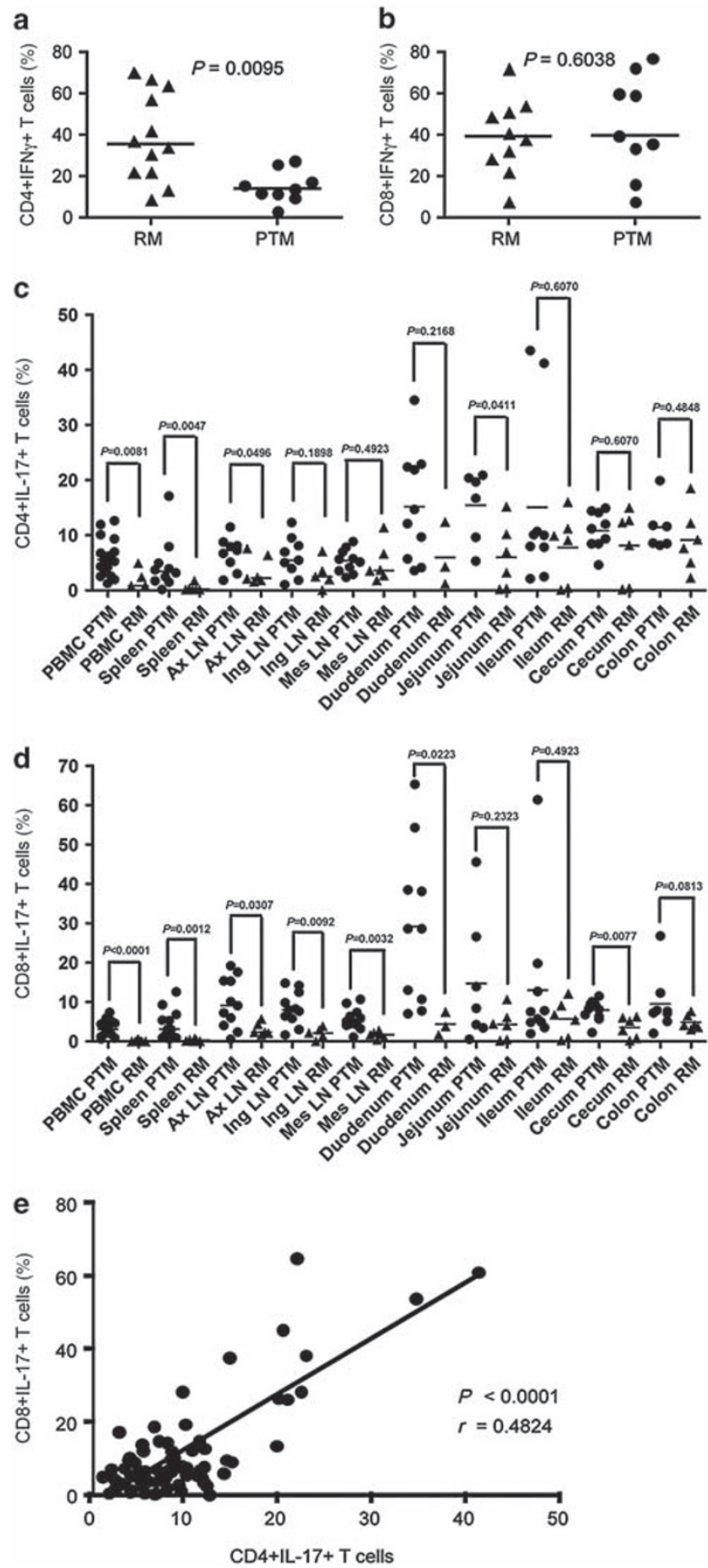

Figure 3 High frequencies of T cells from uninfected pigtail macaques (PTMs) produce IL-17 compared with rhesus macaques (RMs).

(a) Frequencies of CD4 + interferon $\gamma$ (IFN $\gamma$ )-producing T cells in blood; (b) frequencies of CD8 + IFN $\gamma$-producing T cells in blood; (c) frequencies of CD4 + IL-17-producing T cells in blood and necropsy tissues;

(d) frequencies of CD8 + IL-17-producing T cells in blood and necropsy tissues; and (e) frequencies of CD4 + vs. CD8 + IL-17-producing T cells in blood of PTM. Correlation ( $r$ ) determined by Spearman's rank correlation. All samples were stimulated with phorbol myristate acetate and ionomycin. PTM, circles and RM, triangles. Horizontal bars indicate median. $P$-values from Mann-Whitney $U$-test. Ax $L N$, axillary lymph node; Ing LN, inguinal LN; Mes LN, mesenteric LN; PBMC, peripheral blood mononuclear cell. 
Furthermore, we found that there is a significant, direct correlation between the frequency of IL-17 production between CD4+ and CD8 + T cells in PTM (Spearman correlation: $r=0.4824$, $P<0.0001$; Figure 3e). These data suggest that the same antigens may drive expansion of both IL-17-producing CD4 + and CD8 + T cells in PTM. Given the known antigen-specificity of IL-17producing $\mathrm{CD} 4 \mathrm{~T}$ cells, ${ }^{65}$ these observations raise the possibility that response to bacterial and fungal antigen may be one of the underlying factors involved in the high frequencies of IL-17producing, activated memory T cells in PTM.

After observing the surprisingly high frequency of IL-17-producing $\mathrm{T}$ cells in peripheral blood, we determined the frequency of IL-17-producing T cells in multiple tissues from PTM and RM. We found CD4 + IL-17+ T cells in all tissues of PTM, with an average of $4.67 \%( \pm 4.9)$ in spleen, $6.7 \%( \pm 3.0)$ in axillary $L N$, $6.03 \%( \pm 3.6)$ in inguinal $\mathrm{LN}, 5.33 \%( \pm 2.1)$ in mesenteric $\mathrm{LN}$, $15.16 \%( \pm 10.1)$ in duodenum, $14.45 \%( \pm 6.5)$ in jejunum, $15.45 \%$ $( \pm 6.5)$ in ileum, $10.83 \%( \pm 3.4)$ in cecum, and $11.423 \%( \pm 4.5)$ in colon (Figure 3c). In comparison, the average frequency of IL-17-producing CD4 + T cells in RM was $0.47 \%( \pm 0.6)$ in spleen, $3.63 \%( \pm 2.62)$ in axillary $\mathrm{LN}, 3.01 \%( \pm 2.6)$ in inguinal $\mathrm{LN}, 4.96 \%( \pm 3.6)$ in mesenteric LN, 5.96\% $( \pm 5.8)$ in duodenum, $6.02 \%( \pm 6.0)$ in jejunum, $7.77 \%( \pm 6.3)$ in ileum, $8.12 \%( \pm 6.5)$ in cecum, and $9.13 \%( \pm 5.7)$ in colon (Figure 3c). Although the only significant difference between CD $4+$ IL- $17+$ T cells in gut tissues of PTM and RM was in jejunum $(P=0.0411)$, significant differences were observed in spleen $(P=0.0047)$ and axillary LN $(P=0.0496)$, which indicates that bacteria-specific IL-17-producing CD4 + T cells are found at higher levels in the periphery than typically observed in RMs or humans (Figure 3c).9,19,65,66

Similarly, high frequencies of IL-17-producing CD8 + $\mathrm{T}$ cells were observed in PTM, with an average of $4.27 \%$ $( \pm 3.8)$ in spleen, $9.72 \%( \pm 6.4)$ in axillary LN, $8.38 \%( \pm 4.2)$ in inguinal LN, $5.59 \%( \pm 2.7)$ in mesenteric LN, $29.19 \%$ $( \pm 20.2)$ in duodenum, $14.68 \%( \pm 16.2)$ in jejunum, $13.03 \%$ $( \pm 17.8)$ in ileum, $7.97 \%( \pm 2.6)$ in cecum, and $9.53 \%( \pm 7.5)$ in colon (Figure 3d). In comparison, RM had very low levels of IL-17-producing CD8 $+\mathrm{T}$ cells, with an average of $0.30 \%( \pm 0.3)$ in spleen, $3.07 \%( \pm 1.6)$ in axillary $\mathrm{LN}, 2.07 \%$ $( \pm 1.5)$ in inguinal $\mathrm{LN}, 1.62 \%( \pm 0.8)$ in mesenteric $\mathrm{LN}$, $4.32 \%( \pm 2.9)$ in duodenum, $4.32 \%( \pm 3.9)$ in jejunum, $5.74 \%$ $( \pm 4.6)$ in ileum, $3.54 \%( \pm 2.6)$ in cecum, and $4.83 \%( \pm 1.8)$ in colon (Figure 3d). As expected, given the low levels of CD8 + IL-17 + T cells in RM, there was a significant difference between PTM and RM for many tissues in addition to blood, including spleen $(P=0.0012)$, axillary LN $(P=0.0307)$, inguinal LN $(P=0.0092)$, mesenteric LN $(P=0.0032)$, duodenum $(P=0.0223)$, and cecum $(P=0.0077)$, again with the most differences being observed in peripheral and lymphoid tissues (Figure 3d). Of note, similar to CD4 + CCR $5+\mathrm{T}$ cells (Figure 2), the small intestine (duodenum, jejunum, and in some animals in ileum) had the highest frequency of IL-17producing T cells in PTM (Supplementary Tables 4 and 5). Taken together, these data highlight the differences between anatomical sites of the GI tract and show the importance of studying multiple tissues to understand the dynamic nature of mucosal immune compartments more completely.

\section{Uninfected PTMs have high levels of LPS in plasma and increased permeability of the small intestine in comparison with RMs and humans}

Given the presence of antigens (that is, bacteria and fungi) typically targeted by Th17 cells and the increased frequency of IL-17-producing T cells in PTM, we hypothesized that a contributing cause of the increased T-cell activation and high frequencies of IL-17-producing T cells may be microbial translocation. To evaluate the potential systemic dissemination of translocated microbial products in PTMs, we measured plasma levels of LPS that is used as a quantitative indicator of microbial translocation. ${ }^{25,36-41}$ We found that PTMs had higher levels of plasma LPS, with an average of $45.33 \mathrm{pg} \mathrm{ml}^{-1}$ of LPS in the plasma $( \pm 12.6)$, as compared with $\mathrm{RM}$, with an average of $19.21 \mathrm{pg} \mathrm{ml}^{-1}$ of LPS $( \pm 13.3, P=0.0013)$, or with humans, with an average of $20.32 \mathrm{pg} \mathrm{ml}^{-1}$ of LPS $( \pm 15.9, P=0.0014$; Figure 4a). To assess whether the increased LPS was associated with damage to the GI tract, we measured small intestine permeability using a clinically used test based on saccharide permeability that measures the ratio of lactulose to mannitol after saccharide solution ingestion. ${ }^{67,68}$ PTMs showed higher gut permeability, with an average lactulose/mannitol ratio of $0.088( \pm 0.03)$ compared with humans, with an average lactulose/ mannitol ratio of 0.017 ( \pm 0.008$), P=0.0005$ (Figure $4 \mathbf{b}$ ). Hence, one of the potential causes of the increased frequencies of activated and IL-17-producing T cells in PTMs may be microbial products that translocate through a permeable GI tract.
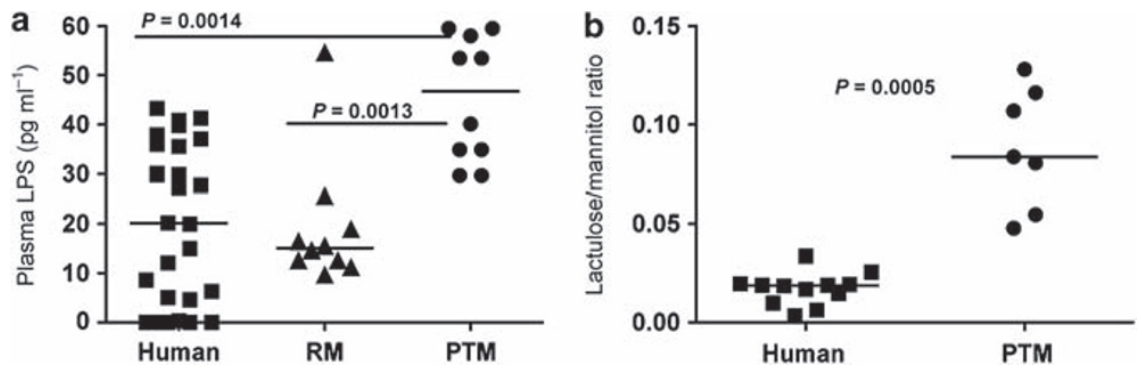

Figure 4 Plasma lipopolysaccharide (LPS) and lactulose to mannitol ratio indicate microbial translocation and gut permeability in uninfected pigtail macaques (PTMs). (a) Plasma LPS levels. (b) Lactulose to manitol ratio. PTM, circles, rhesus macaques (RMs), triangles, humans, squares. Horizontal bars indicate median. $P$-values from Mann-Whitney $U$-test. 

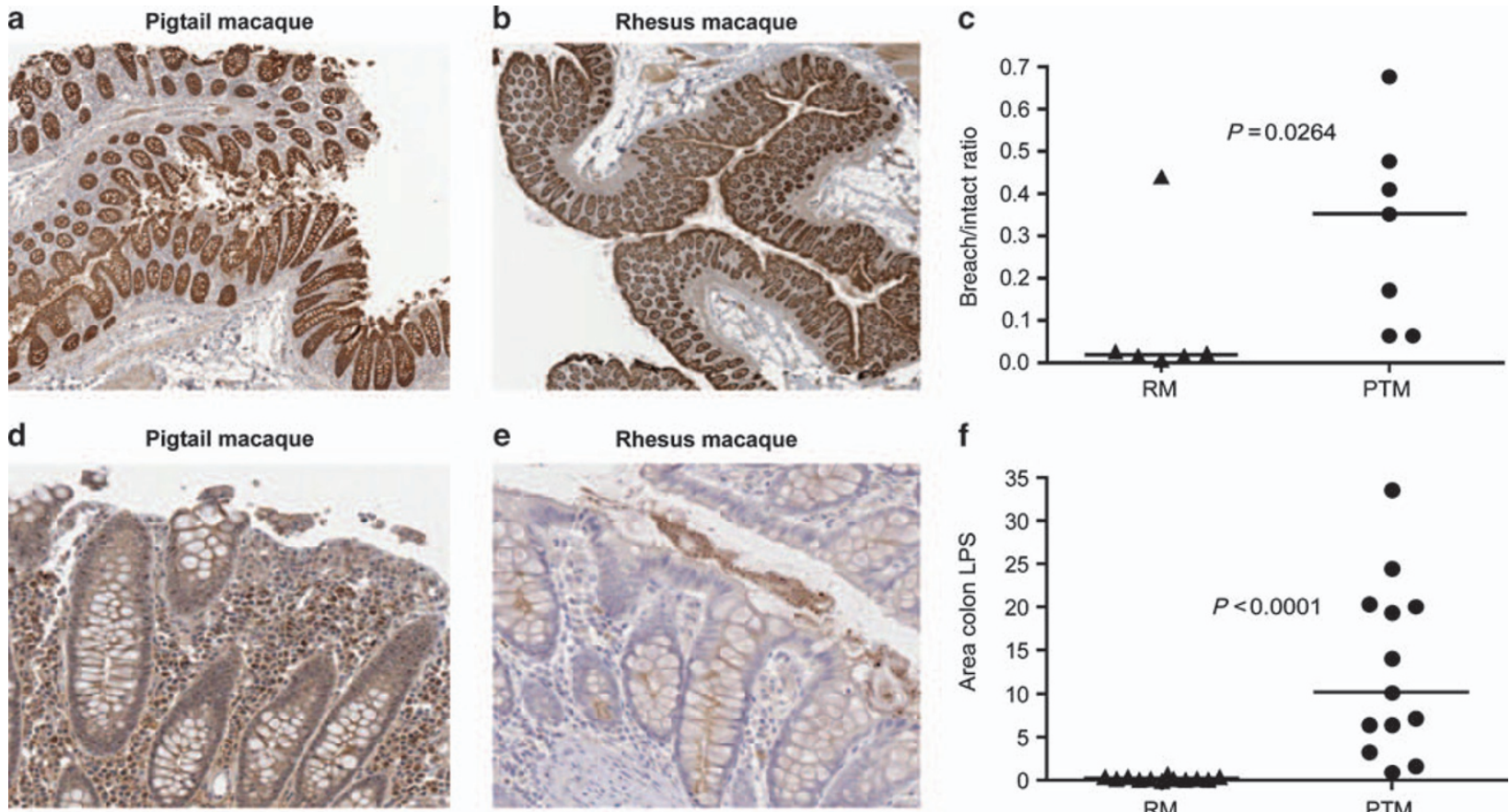

f

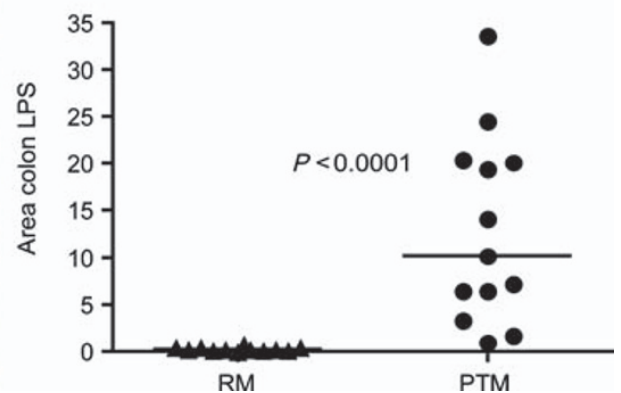

Figure 5 Uninfected pigtail macaques (PTMs) have increased damage to the tight epithelial barrier and high levels of lipopolysaccharide (LPS) in the lamina propria of the colon. (a) Representative claudin-3 staining (brown) in colon of PTM; (b) representative claudin-3 staining (brown) in colon of rhesus macaques (RMs); (c) ratio of measurements of breached epithelial (no claudin) to intact epithelial (claudin) cells in either PTM or RM; (d) representative LPS staining (brown) in colon of PTM; (e) representative LPS staining (brown) in colon of RM; and (f) area of colon lamina propria LPS measured in PTM and RM. PTM, circles, RM, triangles. Horizontal bars indicate median. P-values from Mann-Whitney U-test.

\section{Uninfected PTMs show increased damage to the gut epithelia and microbial translocation compared with RMs}

To determine the mechanism underlying the permeability of the GI tract and consequent microbial translocation in PTM, both PTMs and RMs were killed, and immunohistochemical (IHC) studies were performed on sections of the GI tract taken at necropsy. Initially, we stained GI tract tissues with antibodies against the tight junction protein, claudin-3, to measure the continuity of the structural barrier of the gut epithelia. This analysis allowed us to determine to what extent breaches and ulcerations in the tight epithelial barrier occur in PTMs. We found that PTMs (Figure 5a) had a much higher frequency of breaches in the epithelium of the colon, as compared with RMs (Figure 5b). To confirm these findings, we used quantitative image analysis to determine the percentage of damaged GI barrier (breach/intact ratio) by measuring the length of the tight epithelial barrier that was not intact (not stained for claudin) over the length of the colon barrier that was intact (stained for claudin). On average, the breach/intact ratio for PTM was $0.303( \pm 0.21)$, compared with $0.017( \pm 0.01)$ for RM, $P=0.0264$ (Figure 5c); hence, a putative mechanism for the observed increase in frequency and extent of diarrhea, intestinal permeability, and microbial translocation in PTM may be the relatively increased preexisting damage to the structural barrier of the GI tract.

To determine whether these breaches in the tight junctions of the epithelium correlated with the observed increase in microbial translocation, we studied the colon sections by IHC using an antibody against the LPS core antigen to directly measure the bacterial products present within the lamina propria (LP). We found that PTMs had increased levels of LPS in the LP of the colon (Figure 5d), as compared with RMs (Figure 5e). Using quantitative image analysis, we determined the percent area of colonic LP that contained LPS and found that on average, LPS accounted for $13.00 \%( \pm 10.01)$ of the LP area in PTM, whereas only $0.274 \%( \pm 0.20)$ of the LP was occupied by LPS in $\mathrm{RM}(P<0.0001$; Figure 5f). These data strongly suggest that a mechanism underlying the increased microbial translocation involves structural damage to the gut epithelium in PTM. Indeed, we found a significant positive correlation between the breach/intact ratio and the area fraction of colon LPS in PTM (Spearman $r=0.6424, P=0.0368$; Figure 6a).

\section{Damage to gut epithelium in uninfected PTMs results in} systemic microbial translocation that correlates with immune activation

To determine the significance of microbial translocation contributing directly to immune activation, we also quantified the amount of immune activation in the colon of PTMs by performing quantitative image analysis of colon sections that were stained with a monoclonal antibody against $\mathrm{MxA}$, a protein produced in response to recent production of type-I IFNs. ${ }^{69,70} \mathrm{We}$ found that on average, $11.55 \%( \pm 11.29)$ of the cellular area in the LP of the colon of PTMs were stained for MxA. The extent of microbial translocation positively correlated with local immune activation in the colon, based on analysis of the fraction of colon LP tissue sections staining for LPS and MxA (Spearman $r=0.7133, P=0.0118$; Figure $\mathbf{6 b}$ ). In an attempt to understand the 

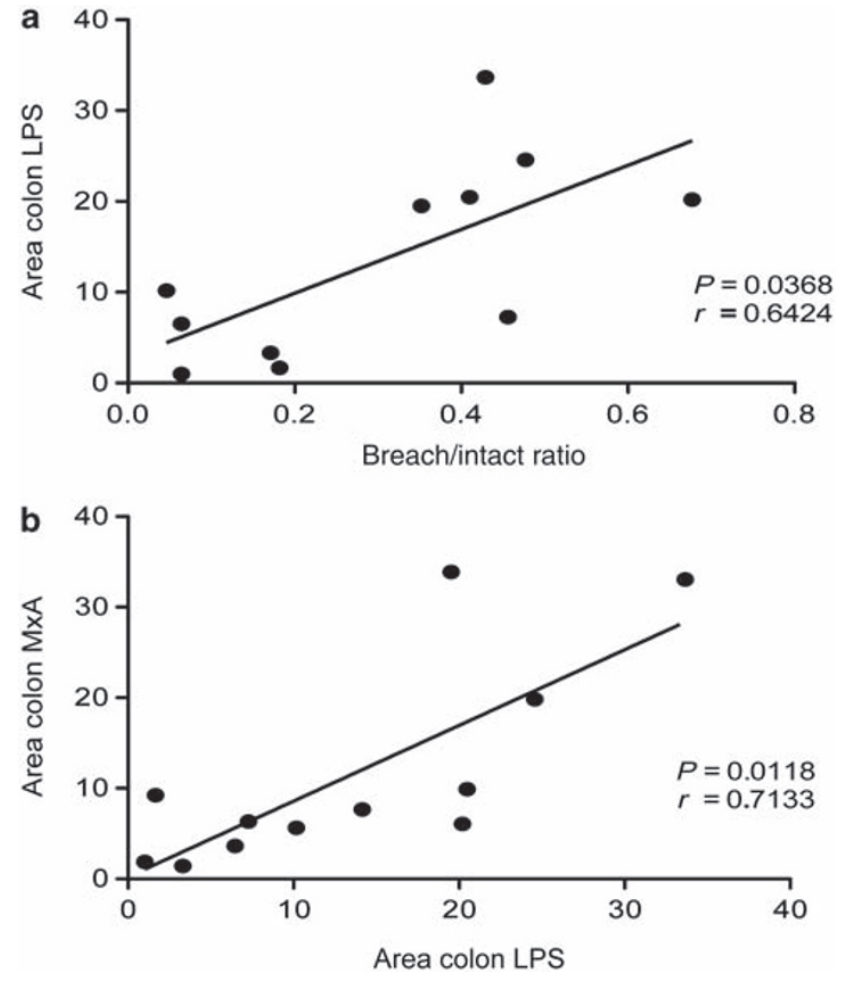

Figure 6 Breaches in the colon correlate with microbial translocation and microbial translocation correlates with immune activation in the colon of uninfected pigtail macaques (PTMs). (a) The breach/intact ratio of the colon, as measured by claudin, significantly correlates with the amount of microbial translocation into the lamina propria of the colon of PTM.

(b) The amount of MxA (a specific type-I interferon-responsive gene) in the colon, as measured by immunohistochemistry staining, significantly correlates with the amount of microbial translocation into the colon of PTM. Correlations determined by Spearman's rank correlation. Lines indicate linear regression. LPS, lipopolysaccharide.

molecular mechanism(s)/events leading to GI epithelial barrier damage in uninfected PTMs, we reasoned that increased enterocyte apoptosis could lead to increased microbial translocation in these animals by altering epithelial barrier homeostasis and function. Thus, we investigated whether the increased damage to the epithelial barrier and resulting microbial translocation that we observe in the colon of PTM may be a result of increased enterocyte cell death by apoptosis. To test this hypothesis, we measured active caspase- 3 by IHC in the colon of PTM and RM and found that, indeed, where colonic epithelial cells were still present, there were higher levels of apoptosis in the enterocytes along the colon cryptal tips in PTM compared with RM (data not shown).

To determine whether the microbial translocation and immune activation that was observed in the colon resulted in systemic consequences, we also used IHC to measure LPS and $\mathrm{MxA}$ in mesenteric $\mathrm{LN}$ that drain the GI tract, as well as in peripheral, axillary LN. With IHC and quantitative image analysis, we found a significant positive correlation between the fractional area of tissue sections staining for LPS in the colon and in the mesenteric LN (Spearman $r=0.5604, P=0.0463$; Figure 7a), as well as between the area staining for MxA in the colon and in the mesenteric LN (Spearman $r=0.6993, P=0.0142$;
Figure $7 \mathbf{b}$ ). Furthermore, there was a significant direct correlation between LPS in mesenteric LNs and LPS in peripheral axillary LNs (Spearman $r=0.6648, P=0.0132$; Figure 7c); as well as between MxA in mesenteric LNs and MxA in axillary LNs (Spearman $r=0.6868, P=0.0095$; Figure 7d). These data indicate that translocation of microbial products and the resulting immune activation not only have local consequences within the GI tract but are also associated with systemic dissemination of LPS and systemic immune activation. We established further evidence of systemic microbial translocation by measuring plasma levels of soluble CD14 (sCD14), which is produced by CD14 + monocytes and macrophages after direct LPS stimulation in vivo. ${ }^{36}$ We found an association between LPS in the colon and SCD14 in the plasma (Spearman $r=0.5000, P=0.0819$; Figure 7e), and there is a significant direct correlation between MxA in the colon and sCD14 (Spearman $r=0.7203, P=0.0106$; Figure 7f). Taken together, these data show that when microbial products translocate from the lumen of the colon into the LP, it can result in local and systemic immune activation. Moreover, these data define the degree to which microbial translocation can stimulate the immune system, both locally and systemically, in the absence of ongoing viral replication.

\section{DISCUSSION}

Microbial translocation has been implicated in the deleterious immune activation that characterizes the chronic phase of HIV infection of humans and SIV infection of Asian macaques. However, the degree to which microbial products alone, relative to persistent viral replication, proinflammatory mediators such as cytokines and chemokines, and/or other unknown factors, contribute to immune activation has remained unclear. Using cells and tissue samples from PTMs, animals that have recurring diarrhea and progress to AIDS quickly after SIV infection, we have shown that in the absence of SIV infection, PTMs have increased damage to the GI tract and show increased basal levels of microbial translocation and immune activation. Specifically, we have shown that (i) uninfected PTMs have damage to the tight epithelial barrier of the GI tract; (ii) this damage correlates with translocation of microbial products both locally within the GI tract and systemically in the LN and blood; (iii) the microbial translocation that occurs correlates with immune activation both locally and systemically; and (iv) uninfected PTMs also have higher frequencies of activated and memory $\mathrm{T}$ cells producing IL-17 than do RM. These data indicate that chronic microbial translocation can be associated with increased activation and turnover of lymphocytes that results in decreased frequencies of naïve $\mathrm{T}$ cells, and an increased amount of memory and effector $\mathrm{T}$ cells and proliferating (Ki67+) T cells. Furthermore, these data are supported by the increased frequencies of CCR5 $+\mathrm{T}$ cells in PTM. CCR5 is found only on the surface of memory and effector T cells (not naïve), as it is needed for lymphocytes to migrate to effector sites such as the GI tract. ${ }^{71,72}$ Moreover, CCR5 + CD4 + $\mathrm{T}$ cells are the primary targets for virus replication after SIV/HIV infection. Hence, it is possible that the increased frequency of these cells in PTM may contribute to the more rapid SIV disease progression that we observe in these animals. It is of interest, 
a

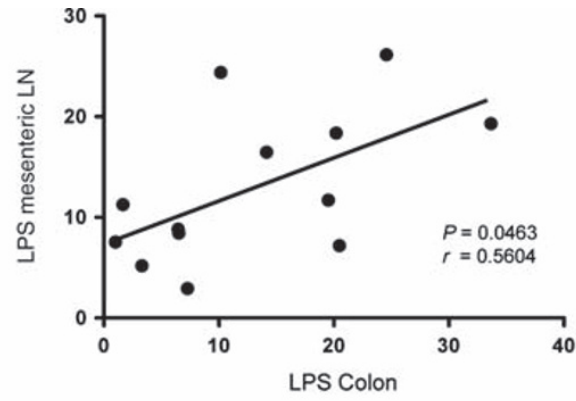

C

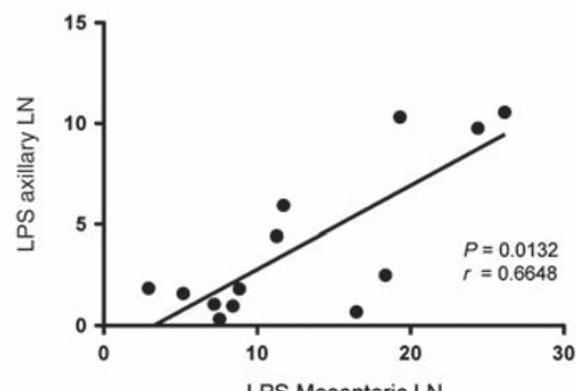

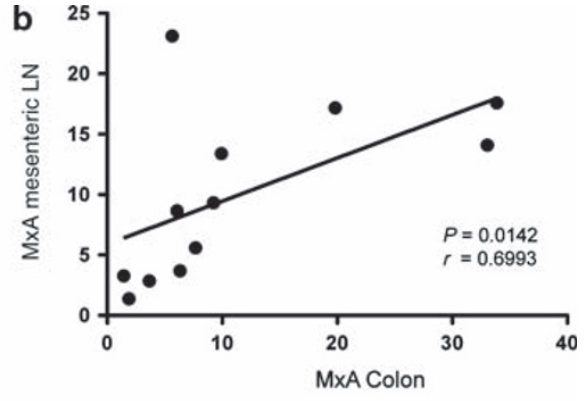

d

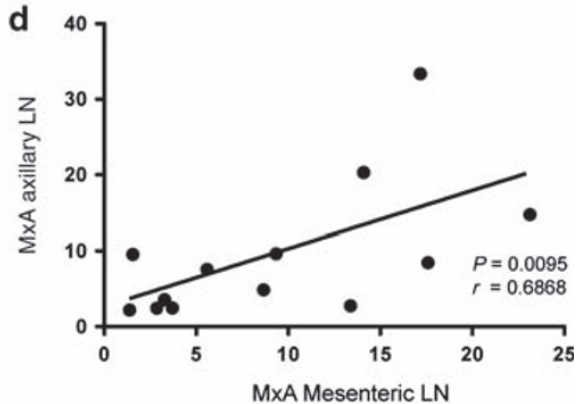

MxA Mesenteric LN

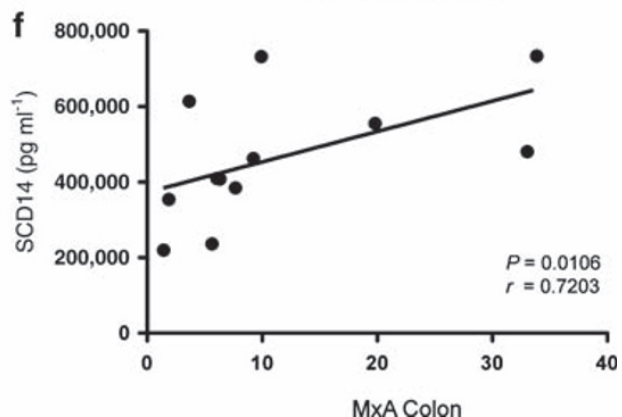

Figure 7 Local microbial translocation and immune activation in the colon results in systemic microbial translocation and immune activation in uninfected pigtail macaques. (a) The amount of lipopolysaccharide (LPS) measured in the colon significantly correlates with LPS in the mesenteric lymph node (LN). (b) The amount of MxA in the colon significantly correlates with the amount of MxA in the mesenteric LN. (c) LPS in the mesenteric LN significantly correlates with LPS in the axillary LN. (d) MxA in the mesenteric LN significantly correlates with MxA in the axillary LN. (e) LPS in the colon is associated with soluble CD14 (sCD14) in plasma. (f) MxA in the colon significantly correlates with sCD14 in plasma. Correlations determined by Spearman's rank correlation. Lines indicate linear regression.

however, that the increased number of target cells do not seem to affect transmission or infection, as previous studies using PTM have shown that these animals get infected at the same rate as RM, with similar peak and set-point viremia, despite inoculation size or infection route (that is, mucosal infection vs. intravenous infection). ${ }^{55,73}$ However, the increased immunological state and number of target cells in PTM provide a plausible mechanism for the more rapid progression to AIDS by destroying more activated $\mathrm{CD} 4+\mathrm{T}$ cells through both viral-mediated and bystander cell death, with a reduced capacity to regenerate these important effector populations because of the reduced naïve T-cell pool. Finally, given the differential frequencies of CCR $5+$ CD $4+$ $\mathrm{T}$ cells in different sites, with the largest frequency of these targets for SIV replication in the small intestine, these data highlight the importance of examining multiple tissues when considering effector sites in the context of mucosal immunology.

Further evidence that microbial translocation is causing the immune activation and high frequencies of memory and activated $\mathrm{T}$ cells, which we observed, is the functionality of $\mathrm{T}$ cells in PTM. Although PTM actually had lower frequencies of Th1
T cells that produce IFN $\gamma$ compared with RM, these animals had very high frequencies of IL-17-producing T cells in all tissues. IL-17 production by T cells is typically in response to bacterial and fungal antigens; ${ }^{65,74}$ thus, it is likely that the translocation of bacterial products that we observe in PTM contributes to the increased IL-17 production by T cells. These data also have implications for viral pathogenesis, as even though IL-17 production is important for immunity against extracellular pathogens, these cells typically lack antiviral mediators that may contribute to lack of control of viremia after SIV infection, while providing excellent targets for SIV infection, as these cells can be infected in vivo. ${ }^{65,74}$ Moreover, these cells have been shown to be preferentially lost from the GI tract in progressive HIV/SIV infections and in imbalance with Treg CD4 + T cells. ${ }^{9,65,74}$ Of interest were the high frequencies of IL-17-producing CD8 + T cells. IL-17 production is generally associated with CD4+ T cells (Th17 cells); ${ }^{4-8}$ however, more recently, several studies have addressed CD8 + T cells that produce IL-17 (Tc17 cells), particularly in the context of chronic inflammation. ${ }^{75-78}$ Of relevance, Kader et al. ${ }^{76}$ recently showed that SIV progression 
in RM is associated with dysregulation of both Th17 and Tc17 cells. Here, we show that not only do high frequencies of CD8 + $\mathrm{T}$ cells produce IL-17 in all tissues but also that there is a correlation between the frequencies of IL-17-producing CD4 + T cells and CD8 + T cells, which implies that these cells may be stimulated by the same types of antigens. IL-17-producing T cells can, themselves, contribute to mucosal health by providing cytokines such as IL-21, which is important for enterocyte homeostasis and induction of antibacterial defensins. ${ }^{13,15,79}$ However, these $\mathrm{T}$ cells are also proinflammatory, suggesting that a delicate balance needs to be achieved to maintain mucosal integrity and health and prevent disease, which was shown by Favre et al. ${ }^{9}$ in SIV infection of PTM. Indeed, there is an association between increased frequencies of Th17 cells and inflamed mucosa in inflammatory conditions such as Crohn's disease and ulcerative colitis. ${ }^{13,19,20,22,23}$ Therefore, in the case of PTM, microbial translocation could likely be causing excessive skewing of $\mathrm{T}$ cells toward a Th17 phenotype, which may, in turn, contribute to immune activation and higher frequencies of memory $\mathrm{T}$ cells by enhancing an ongoing proinflammatory state in PTM.

This study highlights the importance of the consequences of damage to the GI tract and the resulting microbial translocation and consequent stimulation to the immune system, particularly as it was conducted in SIV naïve animals in the absence of the effects of chronic virus infection. Microbial translocation has been well characterized in HIV and SIV infections, and clearly it is a factor contributing to the systemic, chronic immune activation that ultimately leads to disease progression and AIDS. ${ }^{25,36-41}$ However, to our knowledge, this is the first assessment of the extent to which damage to the GI tract and microbial translocation results in both local and systemic effects in the absence of chronic virus replication or chronic proinflammatory diseases. Not surprisingly, although this study shows the extent to which microbial translocation results in immune activation, it also clearly shows that microbial translocation and immune activation alone, in the absence of SIV or HIV replication, is not sufficient to cause AIDS. Thus, in the case of SIV/HIV infection, although increased microbial translocation and immune activation likely have a large role in disease progression, virus replication, proinflammatory responses to the virus, and other contributing factors also are required for progression to AIDS. This study also emphasizes the degree to which dysfunction of the MALT locally results in systemic consequences. Moreover, the increased microbial translocation and consequent immune activation may likely be a mechanistic feature underpinning the increased disease progression rate observed in SIV-uninfected captive PTMs. ${ }^{53,54}$ This is likely due not only to the preexisting damage to the GI tract but rapid progression may also result as a consequence of increased activated CD $4+\mathrm{T}$ cells as target cells for virus replication that exist because of the ongoing immune activation in PTM. Of note, these observations are not isolated to a single colony of PTM, as both diarrhea and rapid SIV progression have been observed in PTMs worldwide (Shiu-Lok Hu, University of Washington and Stephen J Kent, University of Melbourne; personal communication). Given the increased intestinal permeability and microbial translocation that we observed in uninfected PTMs, this model should be well suited for assessing therapeutic interventions such as prebiotics and probiotics, as well as other dietary supplements, in order to reduce microbial translocation and pathogenic consequences of damage to the GI tract. Taken together, these data highlight the importance of microbial translocation in disease progression and suggest that the PTM model is an ideal system to study therapeutic interventions that improve the structural and immunological functions of the GI tract.

\section{METHODS}

Animals and sample collection. For this study, 13 SIV-uninfected PTMs (M. nemestrina) and 10 SIV-uninfected RMs (M. mulatta) were killed and the following tissues were collected: blood, spleen, axillary $\mathrm{LN}$, mesenteric LN, inguinal LN, jejunum, duodenum, ileum, colon, and cecum. Lymphocytes were isolated from LNs and GI tract tissues by grinding tissues through a $0.22 \mu \mathrm{m}$ cell strainer. Lymphocytes were isolated from blood by ficoll gradient centrifugation. Lymphocytes were then viably cryopreserved in fetal bovine serum supplemented with $10 \%$ dimethyl sulfoxide (Sigma, St Louis, MO) and stored in liquid nitrogen. Blood was collected from an additional 12 PTMs and $12 \mathrm{RMs}$ for phenotypical analysis, and lymphocytes were collected as described above. Animals were housed and cared in accordance with the American Association for Accreditation of Laboratory Animal Care standards in AAALAC accredited facilities, and all animal procedures were performed according to the protocols approved by the Institutional Animal Care and Use Committees of the National Institute of Allergy and Infectious Diseases, National Institutes of Health.

Flow cytometry. Multicolor flow cytometric analysis was performed on isolated cells according to standard procedures using human monoclonal antibodies that cross-react with PTM and RM. Predetermined optimal concentrations were used of the following antibodies: antiCD3-Alexa700 (clone SP34-2, BDPharmigen, San Jose, CA), anti-CD8 PacBlue (clone RPA-T8, BDPharmigen), anti-CD4 PE-Cy5.5 (clone OKT4, eBioscience, San Diego, CA), anti-CD4 PE-Cy5.5 (clone L200, BDPharmigen), anti-Ki67 FITC (clone B26, BDPharmigen), anti-CCR5 $\mathrm{PE}$ (clone 3A9, BDPharmigen), Aqua Live/Dead amine dye (Invitrogen, Carlsbad, CA), anti-CD28 ECD (clone 28.2, Beckman Coulter, Brea, CA), anti-CD95 PE-Cy5 (clone DX2, BDPharmigen), anti-CD14 APC (clone M5E2, BDPharmigen), anti-IFN $\gamma$ PE-Cy7 (clone 4S.B3, BDPharmigen), and anti-IL-17 PE (clone eBio64CAP17, eBioscience). All samples were permeabilized and fixed using CytoFix/Perm Kit (BDPharmigen) and intracellularly stained to detect Ki67. For intracellular cytokine staining, cells were stimulated with phorbol myristate acetate $\left(5 \mathrm{ng} \mathrm{ml}^{-1}\right)$ and ionomycin $\left(1 \mu \mathrm{Mml}^{-1}\right)$ for $10-14 \mathrm{~h}$ with brefeldin $\mathrm{A}\left(1 \mu \mathrm{g} \mathrm{ml}^{-1}\right)$ added after $1 \mathrm{~h}$ of stimulation. Flow cytometric acquisition was performed on at least 100,000 lymphocytes on a BD FACSAriaII cytometer driven by the FACS DiVa software (version 6.0; BDPharmigen). Analysis of the acquired data was performed using FlowJo software (version 8.8.4; TreeStar, Ashland, OR). We used a threshold of 200 collected events for all analysis of specific cell subsets. Memoryeffector T cells were determined by gating on both CD28 + CD95 + and CD28 - subsets, whereas CD28 + CD95 - T cells were determined to be naïve cells.

Immunohistochemistry. Immunohistochemical staining was performed as previously described.$^{80}$ In brief, unselected specimens of tissues of interest were obtained at necropsy, fixed in $4 \%$ araformaldehyde, and paraffin embedded. Immunohistochemistry was performed using a biotin-free polymer approach (MACH-3; Biocare Medical, Concord, CA) on $5-\mu \mathrm{m}$ tissue sections mounted on glass slides that were dewaxed and rehydrated with double-distilled water. Antigen 
retrieval was performed by heating sections in $1 \times$ DIVA Decloaker reagent (Biocare Medical) in a pressure cooker (Biocare Medical) followed by cooling to room temperature. All slides were stained using the intelliPATH FLX autostaining system (Biocare Medical) according to the experimentally determined optimal conditions. This included blocking tissues with sniper blocking reagent (Biocare Medical) for $10 \mathrm{~min}$ followed by an additional blocking step with 1,3,5-trinitrobenzene (TNB) containing $2 \%$ blocking reagent and $2 \%$ goat serum (Gibco/Invitrogen, Carlsbad, CA) for $10 \mathrm{~min}$ at room temperature. Endogenous peroxidase was blocked with $1.5 \%(\mathrm{v} / \mathrm{v}) \mathrm{H}_{2} \mathrm{O}_{2}$ in Tris-buffered saline ( $\mathrm{pH}$ 7.4). Primary antibodies were diluted in TNB containing $2 \%$ blocking reagent and $2 \%$ goat serum for $1 \mathrm{~h}$ at room temperature. Mouse or rabbit MACH-3 secondary polymer systems (Biocare Medical) were applied for $20 \mathrm{~min}$ each. Sections were developed with ImmPACT DAB chromogen (Vector Laboratories, Burlingame, CA), counterstained with hematoxylin, and mounted in Permount (Fisher Scientific, Waltham, MA). All stained slides were scanned at high magnification $(\times 400)$ using the ScanScope CS System (Aperio Technologies, Vista, CA) yielding high-resolution data from the entire tissue section. Primary antibodies used were mouse anti-LPS core (clone WN1 222-5; Hycult, Uden, The Netherlands or provided by Dr Robin Barclay), polyclonal rabbit anti-Claudin-3 (Labvision, Fremont, CA) and anti-MxA (clone M143; from Drs Otto Haller and Georg Kochs, Department of Virology, Universitätsklinikum Freiburg, Germany).

Quantitative image analysis. To quantify the extent of epithelial barrier damage in the colon (claudin-3), microbial translocation into the LP and lymph nodes (LPS), and immune activation in the colon and LN (MxA), 5- $\mu \mathrm{m}$ thick sections were cut from paraffin blocks of unselected colon tissue obtained at necropsy and stained with either monoclonal antibody against LPS or polyclonal antibody for claudin3 and counterstained with hematoxylin. High-power $(\times 400)$ whole tissue scans were obtained using an Aperio ScanScope, as described above and imported into Photoshop CS3 (Adobe Systems, Mountain View, California). Images were manually trimmed to remove the submucosae, muscularis, and residual luminal content, leaving only the LP mucosae to analyze. The percent area of the LP stained for LPS and MxA was determined in essence, as previously described using Photoshop CS3 tools with plug-ins from Reindeer Graphics. ${ }^{80,81} \mathrm{MxA}$ was calculated as the area fraction of the cellular LP, whereas LPS was calculated as the area fraction of the entire LP. The percent area of LN staining for LPS was determined from whole LN scans as given above, but without the need to trim the image. The proportion of the epithelial barrier that was damaged during SIV infection was first determined by manually tracing (in green) the area of the lumen-GI epithelial tract interface that had no claudin-3-staining epithelial cells using the brush tool in Photoshop CS3. The remaining claudin3-staining intact epithelial cell regions were then manually traced (in pink). The breach: intact ratio was calculated by determining the proportion of the image that was green (lack of claudin-3 stain) divided by the epithelial surface area that was intact (pink) using plug-in tools from Reindeer Graphics.

Plasma LPS and sCD14 levels. To determine plasma LPS levels, we diluted plasma samples to $20 \%$ with endoxtoxin-free water and then heated them to $70^{\circ} \mathrm{C}$ for $10 \mathrm{~min}$ to inactivate plasma proteins. We then quantified plasma LPS with a commercially available Limulus Amebocyte assay (Cambrex, East Rutherford, NJ) according to the manufacturer's protocol. Each sample was run in duplicate and background (water control) was subtracted.

To determine plasma sCD14 levels, we diluted plasma samples in the ratio 1:200 with dilution buffer and performed enzyme-linked immunosorbent assay according to the manufacturer's protocol (R \& D Systems, Minneapolis, MN). Each sample was run in duplicate and background (water control) was subtracted.
Statistical analysis. We performed Spearman's rank correlation, linear regression, and Mann-Whitney $U$-tests using Prism 5.0 software (Prism5 for Mac, GraphPad Software, Inc.).

SUPPLEMENTARY MATERIAL is linked to the online version of the paper at http://www.nature.com/mi

\section{ACKNOWLEDGMENTS}

We would like to acknowledge JoAnne Swerczek, Heather CroniseSantis, Richard Herbert, and all the veterinary staff at the $\mathrm{NIH}$ animal center. We would like to thank the Bad Boys of Cleveland (BBC) for advice and helpful discussions. We would like to thank Jon Meddings, Marie Claire Arrieta, and Kyung Park at the University of Alberta and Coreen Beaumier at Walter Reed Medical center for technical support. Judith A Briant is funded by the Howard Hughes Medical Institute Research Scholars Program. These studies were supported by the Intramural National Institute of Allergy and Infectious Diseases, US National Institutes of Health program. This project has also been funded in part with federal funds from the National Cancer Institute, National Institutes of Health, under Contract No. HHSN261200800001E.

\section{DISCLAIMER}

The content of this publication does not necessarily reflect the views or policies of the Department of Health and Human Services, nor does its mention of trade names, commercial products, or organizations imply endorsement by the U.S. government.

\section{DISCLOSURE}

The authors declared no conflict of interest.

(C) 2010 Society for Mucosal Immunology

\section{REFERENCES}

1. Boirivant, M., Amendola, A. \& Butera, A. Intestinal microflora and immunoregulation. Mucosal. Immunol. 1 (Suppl 1), S47-S49 (2008).

2. Turner, J.R. Intestinal mucosal barrier function in health and disease. Nat Rev. Immunol. 9, 799-809 (2009).

3. Baumgart, D.C. \& Dignass, A.U. Intestinal barrier function. Curr. Opin. Clin. Nutr. Metab. Care 5, 685-694 (2002).

4. Aggarwal, S., Ghilardi, N., Xie, M.H., de Sauvage, F.J. \& Gurney, A.L. Interleukin-23 promotes a distinct CD4 T cell activation state characterized by the production of interleukin-17. J. Biol. Chem. 278, 1910-1914 (2003).

5. Langrish, C.L. et al. IL-23 drives a pathogenic T cell population that induces autoimmune inflammation. J. Exp. Med. 201, 233-240 (2005).

6. Harrington, L.E. et al. Interleukin 17-producing CD4+ effector T cells develop via a lineage distinct from the Thelper type 1 and 2 lineages. Nat. Immunol. 6, 1123-1132 (2005).

7. Park, $H$. et al. A distinct lineage of CD4 T cells regulates tissue inflammation by producing interleukin 17. Nat. Immunol. 6, 1133-1141 (2005).

8. Steinman, L. A brief history of $T(H) 17$, the first major revision in the $T(H) 1 /$ $\mathrm{T}(\mathrm{H}) 2$ hypothesis of $\mathrm{T}$ cell-mediated tissue damage. Nat. Med. 13, 139-145 (2007).

9. Favre, D. et al. Critical loss of the balance between Th17 and T regulatory cell populations in pathogenic SIV infection. PLoS. Pathog. 5, e1000295 (2009).

10. Maloy, K.J. \& Kullberg, M.C. IL-23 and Th17 cytokines in intestinal homeostasis. Mucosal. Immunol. 1, 339-349 (2008).

11. Huang, W., Na, L., Fidel, P.L. \& Schwarzenberger, P. Requirement of interleukin-17A for systemic anti-Candida albicans host defense in mice. J. Infect. Dis. 190, 624-631 (2004).

12. Chung, D.R. et al. CD4+ T cells mediate abscess formation in intraabdominal sepsis by an IL-17-dependent mechanism. J. Immunol. 170, 1958-1963 (2003)

13. Brand, S. et al. IL-22 is increased in active Crohn's disease and promotes proinflammatory gene expression and intestinal epithelial cell migration. Am. J. Physiol. Gastrointest. Liver Physiol. 290, G827-G838 (2006).

14. Sugimoto, K. et al. IL-22 ameliorates intestinal inflammation in a mouse model of ulcerative colitis. J. Clin. Invest. 118, 534-544 (2008). 
15. Chen, Y. et al. Stimulation of airway mucin gene expression by interleukin (IL)-17 through IL-6 paracrine/autocrine loop. J. Biol. Chem. 278, 17036-17043 (2003).

16. Wu, Q. et al. IL-23-dependent IL-17 production is essential in neutrophil recruitment and activity in mouse lung defense against respiratory Mycoplasma pneumoniae infection. Microbes Infect. 9, 78-86 (2007).

17. Zelante, T. et al. IL-23 and the Th17 pathway promote inflammation and impair antifungal immune resistance. Eur. J. Immunol. 37, 2695-2706 (2007).

18. Kleinschek, M.A. et al. IL-23 enhances the inflammatory cell response in Cryptococcus neoformans infection and induces a cytokine pattern distinct from IL-12. J. Immunol. 176, 1098-1106 (2006).

19. Annunziato, F. et al. Phenotypic and functional features of human Th17 cells. J. Exp. Med. 204, 1849-1861 (2007).

20. Fujino, S. et al. Increased expression of interleukin 17 in inflammatory bowel disease. Gut 52, 65-70 (2003).

21. Collins, S.M., Vallance, B., Barbara, G. \& Borgaonkar, M. Putative inflammatory and immunological mechanisms in functional bowel disorders. Baillieres Best Pract. Res. Clin. Gastroenterol. 13, 429-436 (1999).

22. Kobayashi, T. et al. IL23 differentially regulates the Th1/Th17 balance in ulcerative colitis and Crohn's disease. Gut 57, 1682-1689 (2008).

23. Monteleone, G., Fina, D., Caruso, R. \& Pallone, F. New mediators of immunity and inflammation in inflammatory bowel disease. Curr. Opin. Gastroenterol. 22, 361-364 (2006).

24. Brenchley, J.M. et al. Preferential infection shortens the life span of human immunodeficiency virus-specific CD4+ T cells in vivo. J. Virol. 80, 68016809 (2006).

25. Brenchley, J.M., Price, D.A. \& Douek, D.C. HIV disease: fallout from a mucosal catastrophe? Nat. Immunol. 7, 235-239 (2006).

26. Gordon, S.N. et al. Severe depletion of mucosal CD4+ T cells in AIDS-free simian immunodeficiency virus-infected sooty mangabeys. J. Immunol. 179, 3026-3034 (2007).

27. Veazey, R.S. et al. Identifying the target cell in primary simian immunodeficiency virus (SIV) infection: highly activated memory CD4+ T cells are rapidly eliminated in early SIV infection in vivo. J. Virol. 74, 57-64 (2000).

28. Guadalupe, M. et al. Severe CD4+ T-cell depletion in gut lymphoid tissue during primary human immunodeficiency virus type 1 infection and substantial delay in restoration following highly active antiretroviral therapy. J. Virol. 77, 11708-11717 (2003).

29. Pandrea, I.V. et al. Acute loss of intestinal $C D 4+T$ cells is not predictive of simian immunodeficiency virus virulence. J. Immunol. 179, 3035-3046 (2007).

30. Sumpter, B. et al. Correlates of preserved CD4(+) T cell homeostasis during natural, nonpathogenic simian immunodeficiency virus infection of sooty mangabeys: implications for AIDS pathogenesis. J. Immunol. 178, 1680-1691 (2007).

31. Klatt, N.R. et al. Availability of activated CD4+ T cells dictates the level of viremia in naturally SIV-infected sooty mangabeys. J. Clin. Invest. 118, 2039-2049 (2008).

32. Giorgi, J.V. et al. Shorter survival in advanced human immunodeficiency virus type 1 infection is more closely associated with T lymphocyte activation than with plasma virus burden or virus chemokine coreceptor usage. J. Infect. Dis. 179, 859-870 (1999).

33. Paiardini, M. et al. Cell-cycle dysregulation in the immunopathogenesis of AIDS. Immunol. Res. 29, 253-268 (2004).

34. Paiardini, M. et al. Perturbations of cell cycle control in T cells contribute to the different outcomes of simian immunodeficiency virus infection in rhesus macaques and sooty mangabeys. J. Virol. 80, 634-642 (2006).

35. Hurtrel, B. et al. Apoptosis in SIV infection. Cell. Death Differ. 12 (Suppl 1), 979-990 (2005).

36. Brenchley, J.M. et al. Microbial translocation is a cause of systemic immune activation in chronic HIV infection. Nat. Med. 12, 1365-1371 (2006).

37. Jiang, W. et al. Plasma levels of bacterial DNA correlate with immune activation and the magnitude of immune restoration in persons with antiretroviral-treated HIV infection.. J. Infect. Dis. 199, 1177-1185 (2009).

38. Baroncelli, S. et al. Microbial translocation is associated with residual viral replication in HAART-treated HIV+ subjects with <50copies/ml HIV-1 RNA. J. Clin. Virol. 46, 367-370 (2009).

39. Marchetti, G. et al. Microbial translocation is associated with sustained failure in CD4+ T-cell reconstitution in HIV-infected patients on long-term highly active antiretroviral therapy. AIDS 22, 2035-2038 (2008).
40. Ancuta, P. et al. Microbial translocation is associated with increased monocyte activation and dementia in AIDS patients. PLOS ONE 3, e2516 (2008).

41. Papasavvas, E. et al. Delayed loss of control of plasma lipopolysaccharide levels after therapy interruption in chronically HIV-1-infected patients. AIDS 23, 369-375 (2009).

42. Anselmi, A. et al. Immune reconstitution in human immunodeficiency virus type 1-infected children with different virological responses to antiretroviral therapy. Clin. Exp. Immunol. 150, 442-450 (2007).

43. Brenchley, J.M. \& Douek, D.C. HIV infection and the gastrointestinal immune system. Mucosal. Immunol. 1, 23-30 (2008).

44. Li, Q. et al. Simian immunodeficiency virus-induced intestinal cell apoptosis is the underlying mechanism of the regenerative enteropathy of early infection. J. Infect. Dis. 197, 420-429 (2008).

45. Sankaran, S. et al. Rapid onset of intestinal epithelial barrier dysfunction in primary human immunodeficiency virus infection is driven by an imbalance between immune response and mucosal repair and regeneration. J. Virol. 82, 538-545 (2008).

46. Daniel, M.D. et al. Simian models for AIDS. Cancer Detect. Prev. Suppl. 1, 501-507 (1987).

47. Letvin, N.L. Animal models for AIDS. Immunol Today 11, 322-326 (1990).

48. Silvestri, G. AIDS pathogenesis: a tale of two monkeys. J. Med. Primatol. 37 (Suppl 2), 6-12 (2008).

49. Picker, L.J. Immunopathogenesis of acute AIDS virus infection. Curr. Opin. Immunol. 18, 399-405 (2006).

50. Veazey, R.S. et al. Gastrointestinal tract as a major site of CD4+ T cell depletion and viral replication in SIV infection. Science 280, 427-431 (1998).

51. Alcantara, S. et al. Thrombocytopenia is strongly associated with simian AIDS in pigtail macaques. J. Acquir. Immune. Defic. Syndr. 51, 374-379 (2009).

52. Batten, C.J. et al. Comparative evaluation of simian, simian-human, and human immunodeficiency virus infections in the pigtail macaque (Macaca nemestrina) model. AIDS Res. Hum. Retroviruses 22, 580-588 (2006).

53. Stratov, I. et al. Short communication: characteristics of effective immune control of simian/human immunodeficiency virus in pigtail macaques. AIDS Res. Hum. Retroviruses 22, 27-32 (2006).

54. Hirsch, V.M. \& Johnson, P.R. Pathogenic diversity of simian immunodeficiency viruses. Virus Res. 32, 183-203 (1994).

55. Polacino, P. et al. Differential pathogenicity of SHIV infection in pig-tailed and rhesus macaques. J. Med. Primatol. 37 (Suppl 2), 13-23 (2008).

56. Brennan, G., Kozyrev, Y. \& Hu, S.L. TRIMCyp expression in old world primates Macaca nemestrina and Macaca fascicularis. Proc. Natl. Acad. Sci. USA 105, 3569-3574 (2008).

57. Brennan, G., Kozyrev, Y., Kodama, T. \& Hu, S.-L. Novel TRIM5 isoforms expressed by Macaca nemestrina. J. Virol. 81, 12210-12217 (2007).

58. Russell, R.G. et al. Epidemiology and etiology of diarrhea in colony-born Macaca nemestrina. Lab. Anim. Sci. 37, 309-316 (1987).

59. Hukkanen, R.R., Liggitt, H.D., Anderson, D.M. \& Kelley, S.T. Detection of systemic amyloidosis in the pig-tailed macaque (Macaca nemestrina). Comp. Med. 56, 119-127 (2006).

60. Rosenberg, Y.J. et al. Variation in T-lymphocyte activation and susceptibility to SIVPBj-14-induced acute death in macaques. J. Med. Primatol. 20, 206-210 (1991).

61. Brenchley, J.M. et al. CD4+ T cell depletion during all stages of HIV disease occurs predominantly in the gastrointestinal tract. J. Exp. Med. 200, 749-759 (2004).

62. MacDonald, T.T. \& Spencer, J. Ontogeny of the gut-associated lymphoid system in man. Acta. Paediatr. Suppl. 83, 3-5 (1994).

63. Veazey, R.S. et al. Characterization of gut-associated lymphoid tissue (GALT) of normal rhesus macaques. Clin. Immunol. Immunopathol. 82, 230-242 (1997).

64. Mattapallil, J.J. et al. Massive infection and loss of memory CD4+ T cells in multiple tissues during acute SIV infection. Nature 434, 1093-1097 (2005).

65. Brenchley, J.M. et al. Differential Th17 CD4 T-cell depletion in pathogenic and nonpathogenic lentiviral infections. Blood 112, 2826-2835 (2008).

66. Cecchinato, V. et al. Altered balance between Th17 and Th1 cells at mucosal sites predicts AIDS progression in simian immunodeficiency virus-infected macaques. Mucosal. Immunol. 1, 279-288 (2008).

67. Laker, M.F., Bull, H.J. \& Menzies, I.S. Evaluation of mannitol for use as a probe marker of gastrointestinal permeability in man. Eur. J. Clin. Invest. 12, 485-491 (1982). 


\section{ARTICLES}

68. Laker, M.F. \& Menzies, I.S. Increase in human intestinal permeability following ingestion of hypertonic solutions. J. Physiol. 265, 881-894 (1977).

69. Ronni, T., Sareneva, T., Pirhonen, J. \& Julkunen, I. Activation of IFN-alpha, IFN-gamma, MxA, and IFN regulatory factor 1 genes in influenza A virusinfected human peripheral blood mononuclear cells. J. Immunol. 154, 2764-2774 (1995).

70. Haller, O., Staeheli, P. \& Kochs, G. Interferon-induced Mx proteins in antiviral host defense. Biochimie 89, 812-818 (2007).

71. Veazey, R.S. et al. Dynamics of CCR5 expression by CD4+ T cells in lymphoid tissues during simian immunodeficiency virus infection. J. Virol. 74, 11001-11007 (2000).

72. Picker, L.J. et al. IL-15 induces CD4 effector memory T cell production and tissue emigration in nonhuman primates. J. Clin. Invest. 116, 1514-1524 (2006).

73. Ho, O. et al. Pathogenic infection of Macaca nemestrina with a CCR5tropic subtype-C simian-human immunodeficiency virus. Retrovirology 6 , 65 (2009).

74. Sallusto, F. \& Lanzavecchia, A. Human Th17 cells in infection and autoimmunity. Microbes Infect. 11, 620-624 (2009).
75. Yen, H.R. et al. Tc17 CD8 T cells: functional plasticity and subset diversity. J. Immunol. 183, 7161-7168 (2009).

76. Kader, M., Bixler, S., Piatak, M., Lifson, J. \& Mattapallil, J.J. Anti-retroviral therapy fails to restore the severe Th-17: Tc-17 imbalance observed in peripheral blood during simian immunodeficiency virus infection. J. Med. Primatol. 38 (Suppl 1), 32-38 (2009).

77. Huber, M. et al. A Th17-like developmental process leads to CD8(+) Tc17 cells with reduced cytotoxic activity. Eur. J. Immunol. 39, 1716-1725 (2009).

78. Ortega, C. et al. IL-17-producing CD8+ T lymphocytes from psoriasis skin plaques are cytotoxic effector cells that secrete Th17-related cytokines. J. Leukoc. Biol. 86, 435-443 (2009).

79. Sugimoto, K. et al. IL-22 ameliorates intestinal inflammation in a mouse model of ulcerative colitis. J. Clin. Invest. 118, 534-544 (2008).

80. Estes, J.D. et al. Early resolution of acute immune activation and induction of PD-1 in SIV-infected sooty mangabeys distinguishes nonpathogenic from pathogenic infection in rhesus macaques. J. Immunol. 180, 6798-6807 (2008).

81. Estes, J. et al. Collagen deposition limits immune reconstitution in the gut. J. Infect. Dis. 198, 456-464 (2008). 